\title{
Tratamiento farmacológico de las depresiones resistentes
}

RESUMEN: La reciente aparición de nuevos antidepresivos y otros psicofármacos ha ampliado las posibilidades de tratamiento de las depresiones resistentes. Pero cuál es el verdadero significado de depresión resistente al tratamiento. Este artículo realiza una revisión de la definición de éste término y otros como respuesta al tratamiento, falta de respuesta, remisión, respuesta parcial; así como de las estrategias de tratamiento de potenciación, combinación y las posibles ventajas del cambio de antidepresivo.

PALABRAS CLAVE: tratamiento, depresión resistente, algoritmo.
SUMMARY: The new spectre of antidepressant and other drugs has increased the chances of management of treatment-resistant depression. But what is really the meaning of this term. This article reviews the definitions of treatment-resistant depression, treatment response, nonresponse, remission, partial response; and also the strategies of augmentation and combination and the possible advantages of switching to a different monotherapy.

KEY WORDS: treatment, resistant depression, algorithms.

\section{Introducción.-}

No existe aún un consenso entre los distintos autores sobre los criterios necesarios para considerar una depresión como resistente al tratamiento (DRT) (1). Una de las definiciones más estrictas es la de Fink (2), que considera imprescindible la falta de respuesta a la terapia electroconvulsiva (TEC) como criterio de resistencia. En el otro extremo, Feighner (3) considera como resistente a la depresión que no responde a cualquier tipo de tratamiento incluida la TEC. Entre las definiciones más aceptadas se encuentra la de Quitkin (4), que establece como resistente al tratamiento aquel episodio depresivo que no responde a $300 \mathrm{mg}$ de Imipramina u otro antidepresivo tricíclico (ADT) a dosis equivalentes, o $90 \mathrm{mg}$ de Fenelcina, durante 6 semanas. Otros autores como Sovery proponen una definición operativa como la falta de respuesta a dos intentos adecuados con dos clases diferentes de antidepresivos (5). Instrumentos de evaluación como la ATHF (The Antidepressant Treatment History Form) evalúan la correcta dosificación, duración y adherencia al tratamiento como requisitos de imprescindible cumplimiento para poder catalogar a una depresión como DRT (6-10). Usando criterios estrictos de 
DRT, estudios recientes muestran que sólo el 50\% de los episodios depresivos reciben un tratamiento correcto $(6,7)$.

La falta de acuerdo en la definición del concepto es, probablemente, la causa de que los datos de incidencia y prevalencia de la DRT oscilen entre márgenes muy amplios. Fava y Davidson (11) realizaron un estudio basado en la literatura existente entre los años 93 y 95, encontrando tasas de prevalencia que oscilaban entre el 20 y el $40 \%$. Estos autores incluían a respondedores parciales siguiendo la propuesta de Fawcet (12), según la cual estos pacientes deberían iniciar los protocolos de tratamiento de DRT debido al sufrimiento y a la repercusión que la enfermedad ejerce sobre las distintas áreas de su vida $(13,14,15)$.

\section{Conceptos afines}

En el campo de la investigación, los conceptos de remisión, respuesta, respuesta parcial y falta de respuesta, se basan en las escalas de evaluación. La más aceptada en los últimos 40 años ha sido la escala de Hamilton para la depresión (HAM-D) (16).

Aunque podríamos suponer que la remisión de un episodio depresivo fuera equivalente a la ausencia de síntomas, en investigación clínica se considera remisión el hecho de alcanzar un punto de corte de la escala HAM-D de entre 7 y 10 puntos, a pesar de que incluso una puntuación menor de 7 refleja la existencia de síntomas residuales (17).

Se considera respondedor a aquel cuya puntuación de la HAM-D desciende en un $50 \%$ con respecto al valor de inicio $(18,19)$. El problema surge cuando en depresiones graves con una alta puntuación de partida, la cifra final sigue siendo lo suficientemente elevada como para volver a ser incluido en el ensayo. Como alternativa a esta contradicción se apuesta por el uso de la escala de Impresión Clínica Global (CGI), aunque con la desventaja de una menor precisión con respecto a la HAM-D(20).

Los no respondedores quedarían relegados a aquellos en que no se diera un descenso de al menos un $25 \%$ en la HAM-D (21).

\section{Factores que influyen en la resistencia al tratamiento}

En aquellos casos en los que un episodio depresivo no responde a ninguno de los intentos de tratamiento que con mayor frecuencia son usados en la práctica clínica, nos veremos abocados a la administración de fármacos cada vez menos estudiados y por tanto menos seguros. Con el fin de evitar estos tratamientos, a menudo innecesarios, es preciso llevar a cabo un estudio exhaustivo de los casos verificando que realmente nos encontremos ante una DRT.

Una causa frecuente de supuesta resistencia es la existencia de patologías somáticas subyacentes o de trastornos psiquiátricos en comorbilidad $(22,23)$. Son las enfermedades endocrinas y, en concreto, las de los ejes tiroideo y adrenal, las que se asocian con mayor frecuencia. También existe relación con la demencia, accidentes 
vasculares cerebrales, trastornos convulsivos, trastornos del sueño y estados postvirales. Igualmente tratamientos conjuntos con betabloqueantes, calcioantagonistas, esteroides y antihipertensivos podrían interferir en la respuesta terapéutica (24). Algunos autores hacen hincapié en el estado nutritivo, atribuyendo al déficit de algunas sustancias una influencia importante en la falta de respuesta. Las sustancias implicadas con mayor frecuencia son los folatos, vitaminas B1, B6 y B12, Cu y Zn $(25,26,27,28)$.

Con respecto a la comorbilidad psiquiátrica como causa de resistencia, cualquier trastorno, incluido el abuso de sustancias, puede interferir en la evolución del cuadro. Dentro del campo de los trastornos afectivos, algunos autores defienden la importancia de distinguir entre los distintos subtipos para los que establecen equivalencias con fármacos específicos como tratamiento de primera línea. Este sería el caso, entre otras, de la depresión melancólica y los ADT $(29,30,31)$ y de la depresión atípica, donde serían de elección los Inhibidores de la Monoaminooxidasa (IMAOs) $(32,33,34,35)$ o los Inhibidores Selectivos de la Recaptación de la Serotonina (ISRS) (36).

Aunque se acepten arbitrariamente las 4 ó 6 semanas como límite para catalogar a una depresión como resistente, son muchos los casos que mejoran pasado este periodo (37). En realidad, no es inusual que la medicación sea retirada por supuesta ineficacia incluso antes de que se haya cumplido este plazo de tiempo, lo que influye negativamente en la resolución de estos casos, no sólo porque podrían haber respondido de haberse continuado el tratamiento, sino porque ante un episodio depresivo, podemos predecir un peor resultado en relación directa al número de tratamientos previos (38).

Existen otros factores a tener en cuenta, como son el uso de dosis subterapéuticas por parte de los profesionales o el defectuoso cumplimiento de las prescritas por parte de los pacientes. La proporción de pacientes que no utilizan los fármacos como se les prescribió puede llegar al 50\%. Los efectos adversos de los medicamentos, las múltiples tomas al día, el miedo a la dependencia o la falta de una explicación correcta del uso del fármaco, son las causas más frecuentes del incumplimiento (39).

Por último, factores sociales, familiares y psicológicos, suelen influir en la evolución del episodio depresivo. El hecho de indagar e intervenir correctamente sobre estos aspectos evitaría en muchos casos un innecesario cambio de tratamiento (13).

\section{Estrategias en el abordaje de la DRT}

Sustitución por otro antidepresivo

Diversos algoritmos de tratamiento recientes como el TMPA (Texas Medication Algorithm Project) recomiendan el cambio a un antidepresivo de distinta clase tras dos intentos fallidos con antidepresivos de la misma clase $(40,41)$.

El cambio dentro de la misma familia sólo parece ser eficaz en el caso de los ISRS (42). Habitualmente la sustitución directa de un ISRS por otro es bien tolerada, ya que al actuar sobre los mismos receptores no se producen síntomas de discontinuación (43).

Tampoco se precisa de un periodo de superposición de ambos fármacos al cambiar un ISRS por venlafaxina, ya que ambos inhiben la recaptación de serotonina. 
Por otra parte, su administración conjunta podría aumentar el riesgo de efectos cardiovasculares (44) y serotoninérgicos (45) secundarios al aumento de los niveles en sangre de venlafaxina por la inhibición del CYP2D6 por parte de los ISRS.

El cambio de un ISRS por bupropion se indica a menudo cuando los ISRS producen efectos adversos sexuales y no existe comorbilidad con trastornos de ansiedad. $\mathrm{Al}$ actuar por mecanismos distintos, no es conveniente realizar un cambio brusco de uno por otro, recomendándose reducir gradualmente la dosis de ISRS mientras se introduce paulatinamente el bupropion (43).

El uso conjunto de un ISRS y nefazodona puede dar lugar a efectos adversos como un síndrome serotoninérgico, por lo que se recomienda realizar el cambio mediante un periodo de lavado tras la reducción gradual del primero para evitar un síndrome de discontinuación $(43,46,47)$. A pesar de esta recomendación, la asociación de ambos fármacos es una estrategia de tratamiento usada con frecuencia en la DRT.

En lo referente a una sustitución de un ISRS por mirtazapina, al tener mecanismos de acción diferentes y no existir constancia de interacciones graves, es recomendable realizar un cambio secuencial de tratamiento (43).

Para sustituir un ISRS por un ADT, se debe comenzar con una dosis baja del ADT, ya que el uso concomitante con un ISRS o inmediatamente después, aumenta los niveles de ADT por inhibición de su metabolismo. La retirada del ISRS debe ser igualmente gradual para evitar síntomas de discontinuación (43).

Aunque algunos autores cuestionan la eficacia de los ISRS frente a los ADT $(48,49)$, otros aseguran una respuesta favorable a los ISRS en depresiones resistentes a ADT y TEC (50). Al realizar un cambio de un ADT a un ISRS, hay que tener en cuenta que la suspensión brusca del primero puede dar lugar a la aparición de síntomas de rebote colinérgico, por lo que el cambio deberá producirse con un ajuste gradual de ambos fármacos.

Tras finalizar un intento fallido con un IMAO, no debe usarse otro antidepresivo hasta que hayan transcurrido 14 días. La misma medida debe tomarse ante el cambio de un IMAO por otro. Para realizar un cambio de cualquier antidepresivo a un IMAO, debe transcurrir un tiempo cinco veces superior a la vida media de dicho fármaco incluyendo sus metabolitos activos (43).

En cambio, en diversas publicaciones sobre DRT se hace referencia al uso conjunto de IMAOs y ADT $(51,52)$, asociación para la que recomiendan una actitud de estrecha vigilancia. No existen evidencias de esta asociación en ensayos controlados (43).

Si se desea realizar cambios entre venlafaxina, bupropion, nefazodona y mirtazapina, se recomienda un periodo de entrecruzamiento, ya que actúan mediante mecanismos distintos (43).

Estrategias de potenciación

Las estrategias de potenciación (administración de un segundo fármaco que interacciona para amplificar el efecto del primero) y de combinación (suma de los efectos 
de dos antidepresivos), parecen estar afianzándose en los últimos años como las preferidas de los profesionales, frente a los algoritmos que recomiendan que ante una DRT se realice en primer lugar un cambio de antidepresivo y en un segundo paso una potenciación. El motivo principal es que suele considerarse que con un cambio de antidepresivo se estaría retirando un fármaco que podría estar aportando cierto beneficio (53).

En la práctica clínica, parece que estas opciones están siendo las más usadas en respondedores parciales que presentaron pocos efectos secundarios con el antidepresivo. En aquellos en los que los efectos secundarios fueron significativos o la respuesta escasa, sería más adecuado el cambio de antidepresivo(43).

La respuesta a una estrategia de potenciación suele darse a las 3 ó 4 semanas. Se considera conveniente mantener el agente potenciador durante 6 a 9 meses después de que se haya producido la mejoría, procediendo tras este tiempo a una retirada gradual (54).

El agente potenciador más usado es el litio. Existen numerosos ensayos abiertos y de doble ciego que corroboran su eficacia. Thase (55) publicó un estudio con 20 pacientes no respondedores a un ensayo de 12 semanas con imipramina, encontró que un $5 \%$ de la muestra respondía rápidamente a la adicción de litio, mientras que un $65 \%$ lo hacía a las 6 semanas. Se cuestionaba así el efecto starter propuesto por Montigny (56), recomendándose continuar el tratamiento por más tiempo del que se venía realizando. También se ha propuesto la eficacia del litio como antidepresivo frente a placebo, observándose una respuesta dentro de las tres primeras semanas (57). En base a estos resultados se ha criticado a quienes se refieren al efecto del litio como potenciador, proponiéndose el término de asinergia potenciadora (58).

Algunos autores establecen una acción bifásica, recomendando niveles altos para el tratamiento de los trastornos bipolares y bajos (litemias en torno a 0,5) para conseguir un efecto antidepresivo (59).

La adición de litio se ha mostrado eficaz con cualquier familia de antidepresivos $(60,61,62,63)$. El riesgo de toxicidad y los efectos adversos son los responsables de haber mermado la popularidad de esta estrategia (64).

Anticonvulsivantes como carbamacepina, valproato, lamotrigina y gabapentina se usan también como potenciadores, aunque han sido más estudiados en los trastornos bipolares $(54,65,66,67)$.

El efecto de la triyodotironina (T3)se prefiere sobre el de la tiroxina (T4)por su mayor rapidez de acción (68). Esta opción no suele usarse con frecuencia en la práctica clínica debido a los efectos secundarios como el nerviosismo e insomnio, así como a que los estudios publicados están realizados con ADT y no con ISRS $(69,70,71)$.

El uso de antipsicóticos atípicos como risperidona a dosis de 0,5-2 mg (72) y olanzapina a dosis de 5-20 mg $(73,74)$ han mejorado la respuesta en varios estudios en no respondedores a ISRS. Su efecto sobre la ansiedad e irritabilidad les han hecho populares en el tratamiento de la agitación e insomnio refractarios (54).

Existen otras medidas que por haber sido empleadas menos frecuentemente, o por riesgo potencial de toxicidad, resultan menos seguras que las anteriores, debiendo 
aplicarse con mayor precaución y asegurando que el beneficio que aportan sea claramente superior al riesgo que plantean. El triptófano se ha utilizado para potenciar el efecto de los IMAOs, pero aumenta en exceso el riesgo de padecer un síndrome serotoninérgico $(75,76)$. La S-adenosilmetionina, usada para acelerar la respuesta de los ADT (77), no ha sido estudiada en la DRT (54), e incluso se postula que podría aumentar el riesgo de padecer episodios de manía e hipomanía (78). El pindolol se ha usado para potenciar los IMAOs e ISRS $(79,80,81)$, la buspirona con los ISRS $(82,83,84,85)$, agentes dopaminérgicos como la bromocriptina o el pergolide (86) y psicoestimulantes como el metilfenidato (87) también han sido usados como potenciadores. El inositol no ha revelado mayor eficacia que el placebo $(88,89)$, en cuanto a los estrógenos, existen datos anecdóticos en mujeres postmenopáusicas (54). Un estudio doble ciego con una muestra pequeña en el que se usó dehidroepiandrosterona (DHEA) sugiere su eficacia a dosis de $90 \mathrm{mg}$, aunque se precisarían más estudios para confirmarlo (90). Un estudio en el que se añadía folato a fluoxetina, mostró mayor eficacia frente a placebo en mujeres, pero no en hombres (91).

Diversos estudios realizados tanto con prednisona (92) como con dexametasona $(93,94,95)$ en pacientes con hipocortisolemia, así como con antiglucocorticoideos $(96,97,98,99$,$) en hipercortisolémicos revelen resultados prometedores.$

Combinación de antidepresivoLa opción de añadir un segundo antidepresivo precisa de una espera de entre 4-6 semanas para evaluar la respuesta. Esta estrategia debe mantenerse por un periodo de 6 a 9 meses tras el comienzo de la mejoría, momento en el que puede retirarse uno de los fármacos (54).

La asociación más comentada en la literatura, de la que constan varios estudios abiertos, es la de bupropion a dosis de 100-150 mg con un ISRS. Esta asociación puede dar lugar a efectos indeseables como temblor y aparición de ataques de pánico $(100,101)$ a la vez que favorece la reducción de los efectos adversos sexuales que producen los ISRS $(102,103)$. Este mismo efecto beneficioso lo encontramos con la asociación de mirtazapina a los ISRS y ADT (104), opción que por otra parte produce un mayor aumento de peso y sedación (105); y con la nefazodona (106), aunque en este caso tanto este efecto como su beneficio global no está avalado por ensayos clínicos $(54,107,108)$.

En algunos estudios realizados con una pequeña muestra $(109,110)$, se aprecia una respuesta más rápida al añadir un ADT a un ISRS(111). Dado el efecto de estos últimos sobre el CYP2D6, estos estudios se realizan con monitorización y con dosis bajas de ADT, de entre 25 y $75 \mathrm{mg}(54)$.

Debido al riesgo de efectos adversos, son escasos los ensayos controlados sobre la asociación de dos ISRS $(112,113)$ o de un ISRS con venlafaxina $(114,115)$.

La asociación de la reboxetina a un ISRS, opción elegida con frecuencia en la práctica clínica, se muestra como una estrategia eficaz $(116,117)$ y segura $(118)$

\section{Conclusiones}

Mejorar las tasas globales de efectividad al abordar el tratamiento de los trastornos depresivos sigue siendo un reto. A pesar de la aparición de nuevos fármacos 
más eficaces y con menos efectos adversos sigue siendo elevado el porcentaje de pacientes que no mejoran lo suficiente.

En la práctica clínica no parece aceptable aceptar que la mejoría de un episodio depresivo consiste en una reducción de un 50\% de la sintomatología (119). Algoritmos de tratamiento como the Texas Medication Algorithm Project (TMAP) y Sequenced Treatment Alternatives to Relieve Depression (STAR*D) recomiendan apostar por la remisión total de los síntomas y la recuperación de un buen nivel de funcionamiento psicosocial $(120,121)$. Por ello consideran que la mejor estrategia terapéutica es la más agresiva (122).

Los grupos de trabajo encargados de realizar algoritmos de tratamiento fiables y revisar su puesta al día, parecen constituirse como el futuro de una buena práctica clínica asentada sobre la medicina basada en la evidencia (123).

\section{BIBLIOGRAFÍA.}

1. Harold A. Sackeim, Ph. D. : The definition and meaning of treatment-resistant depression. J Clin Psychiatry 2001;62 (suppl 16).

2. Fink M. "Atrial of ECT is essential before a diagnosis of refractory depression is made". In: Refractory depression Amsterdam JD, ed. New York: Raven Press, 1991: 87-92.

3. Feighner JP, Herbstein J, Damlouju N. "Combined MAOI, TCA and direct stimulant therapy of treatment resistant depression”. J Clin Psychiatry 1985; 46: 206-209.

4. Quitkin FM. "The importance of dosage in prescribing antidepressants". Br J psychiatry 1985; 147: 593-7.

5. Souery D, Amsterdam J, de Montigni C, et al. "Treatment resistant depression: methodological overview and operational criteria". Eur Neuropsycopharmacol 1999;9:83-91.

6. Prudic J, Sackeim HA, Devanand DP. “Medication resistance and clinical response to electroconvulsive therapy". Psychiatry Res 1990; 31:287-296.

7. Prudic J, Haskett RF, Mulsant B, et al. "Resistance to antidepressant medications and short term clinical response to ECT". Am J Psychiatry 1996; 153: 985-992.

8. Sackeim HA, Prudic J, Devanand DP, et al. "The impact of medication resistance and continuation pharmacotherapy on relapse following response to ECT in major depression". $J$ Clin Psycopharmacol 1990;10:96-104

9. Mulsant BH, Haskett RF, Prudic J, et al. "Low use of neuroleptic drugs in the treatment of psycotic major depression”. Am J Psychiatry 1997;154:559561.

10. Oquendo MA, Malone KM, Ellis, SP, et al. "Antidepressant treatment is inadequate in patients with major depression who are at the risk for suicidal behavior". Am $j$ Psychiatry 1999; 156:190-194.

11. Fava M, Davidson KG. "Definition and epidemiology of treatment-resistant depression". Psychiatric Clin of North Am. 1996; 19:179-198

12. Fawcet J: "Antidepressants: partial response in chronic depression". Br J Psychiatry 165 (suppl 26) 1993:35-38.

13. Coryell W, Zimmeman M: "Demographic, historical and symptomatic features of the nonmanic psychoses". J Nerv Ment Dis 1986. 174:585-592.

14. Lykouras E, Christodoulou GN, Malliaras D: Type and content of delusions in unipolar psychotic depression. J Affect Disord 1985. 9:249-252 
15. Nelson WH, Khan A, Orr WW: "Delusional depression: Phenomenology, neuroendocrine function, and tricyclic antidepressant response". J Affect Disord 1984. 6: 297-306

16. Carroll BJ, Fielding JM, Blashki TG. "Depression rating scales: a critical view". Arch Gen Psychiatry 1973,28:361-366.

17. Nieremberg AA, Keefe BR, Leslie VC, et al. "Residual symptoms in depressed patients who respond acutely to fluoxetine". J Clin Psychiatry 1999;60:221-225.

18. Frank E, Prien RF,Jarret RB, et al. "Conceptualization and rationale for consensus definitions of terms in major depressive disorder". Arch Gen Psychiatry 1991;48:851-855.

19. Riso Lp, Thase ME, Howland RH, et al. "A prospective test of criteria for response, remission, relapse, recovery, and recurrence in depressed patients treated with cognitive behavioral therapy". J Affect Disord 1997;42:131-142.

20. Guy W: ECDEU Assesment Manual for Psychopharmacology. US Dept Health, Education and Welfare publication (ADM) 76-33, Rockville, Md: National Institute of Mental Health, 1976:218-222.

21. Andrew A. Nieremberg, M.D., and Lindsay M. DeCecco, B.A. "Definitions of antidepressant treatment response, remission, nonresponse, partial response, and other relevant outcomes: a focus on treatment-resistant depression". J Clin Psychiatry 2001;62 (suppl 16)

22. Black DW, Bell S, Hulbert J, et al: "The importance of axis II in patients with major depression: A controlled study". J Affect Disord 1998,14:115-122

23. Hall RC, Gardner ER, Popkin ER, et al: "Unrecognized physical illness prompting psychiatric admission: A prospective study". Am J Psychiatry 1981;138: 629-635

24. Victor I, Reus MD: "Managemente of treatment-resistant unipolar and chronically depressed patients". Psychiatric Clin of North Am. 1996. 19 (2), 201-213.

25. Hansen CR, Malecha M, Mackenzie TB, et al: "Copper and zinc deficiencies in association with depression and neurological findings". Biol Psychiatry 1983;18:395-401

26. Levitt AJ, Joffe RT: "Folate, B12, and life course of depressive illness. Biol Psychiatry" 1989 25:867-872.

27. Lidembaum J, Healton EB, Savage, DG, et al: "Neuropsychiatric disorders caused by cobalamin deficiency in the absence of anemia or macrocytosis". N Engl J Med 1988;26:1720-1728

28. Stewart JW, Harrison W, Quitkin F, et al: "Low B6 levels in depressed outpatients". Biol Psychiatry 1994;19:613-616

29. Danish University Antidepressant Group. "Citalopram: clinical effect profile in comparison with clomipramine. A controlled multicenter study”. Psychopharmacology (Berl) 1986, 90:131-138.

30. Danish University Antidepressant Group. "Paroxetine: a selective serotonin reuptake inhibitor showing better tolerance but weaker antidepressant effect than clomipramine in a controlled multicenter study". J Affect Disord 1990;289-299

31. Perry PJ. "Pharmacotherapy for major depression with melancholic features: relative efficacy of tricyclic versus selective serotonin reuptake inhibitor antidepressants". $J$ Affect Disord 1996;39:1-6.

32. Thase ME, Frank E, Mallinger Ag, et al. "Treatment of imipramine-resistant recurrent depression, 3: efficacy of monoamine oxidase inhibitors". J Clin Psychiatry 1992;53:5-11.

33. Liebowitz MR, Quitkin FM, Stewart JW,et al. "Antidepressant specificity in atypical depression. Arch Gen psychiatry" 1988;45:129-137

34. Quitkin FM, Rifkin A, Klein DF. "Monoamine oxidase inhibitors: a review of antidepressant effectiveness". Arh Gen psychiatry 1979;36:749-760 
35. Thase Me, Trivedi MH, Rush AJ. "MAOIs in the contemporary treatment of depression". Neurophychopharmacology 1995; 12: 185-219

36. Pande AC, Birkett M, Fechner-Bates $\mathrm{S}$ et al. "Fluoxetine versus phenelzine in atypical depression". Biol Psychiatry 1996;40:1017-1020

37. Keller MB, McCullogh JP, Klein DN, et al. "A comparation of nefazodone, the cognitive behavioral-analysis system of psychotherapy, and their combination for the treatment of chronic depression". N Engl J Med 2000;342:1462-1470

38. Amsterdam J, Maislim G, Potter L: "Fluoxetine efficacy in treatment resistant depression". Prog Neurophychopharm Biol Psychiatry 1994;18:143-261

39. Fawcet J, Kravitz HM, Zajacka JM, et al: "CNS stimulant potentiation of monoamine oxidase inhibitors in treatment refractory depression with a monoamine oxidase inhibitor". Psychopharmacol 1991;11:127-132

40. American Psychiatry Association. "Practice Guiedeline for the Treatment of Patients With Major Depressive Disorder (Revision)". Am J Psychiatry 2000;157(suppl 4):1-45

41. Crismon ML, Trivedi M, Pigott TA, et al. "The Texas Medical Algorithm Projet: report of the Texas Consensus Conference Panel on Medication Treatment of Major Depressive Disorder". J Clin Psychiatry 1990;60:142-156

42. Amsterdam J, Maislim G, Potter L: "Fluoxetine efficacy in treatment ressistant depression". Prog Neuropsychopharm Biol Psychiatry 1994,18:143-161

43. Lauren B, Marangell MD: "Switching Antidepressants for Treatment-Resistant Major Depression". J Clin Psychiatry 2001;62 (suppl 18)12-17

44. Lessard E, Yessine MA, Hamelin BA, et al. "Influence of CYP2D6 activity on the disposition and cardiovascular toxicity of the antidepressant agent venlafaxina in humans". Pharmacogenetics 1999;9:435-443

45. Bhatara VS, Magnus RD, Paul KL, et al. "Serotonin syndrome induced by venlafaxine and fluoxetine: a case study in polypharmacy and potential pharmacodinamic and pharmacokinetic mechanims". Ann Pharmacother 1998,32:432-436

46. John L, Perreault MM, Tao T, et al. "Serotonin syndrome associated with nefazodone and paroxetine". Ann Emerg Med, 1997;29:287-289

47. Smith Dl, Wenegrat BG. "A case report of serotonin syndrome associated with combined nefazodone and fluoxetine (letter)". J Clin Psychiatry 2000;61:146

48. Nolen WA, van de Putten JJ, Dijken WA, et al: "Treatment strategy in depression I. Non tricyclic and selective reuptake inhibitors in resistant depression: A double-bind, partial cross-over study on the effects of oxaprotiline and fluvoxamine". Acta Psychiatr Scand 1998,78:668-675.

49. Roose SP: "Selective serotonin reuptake inhibitors in refractory depression". IN Nolen W, Zohar J, Roose S, Amsterdam JD (eds): Refractory depression: Current Strategies and Future Directions. Chichester, John Wiley \& Sons, 1994, pp37-46

50. Beasley CM Jr, Sayler ME, Cunningham GE, et al: "Fluoxetine in tricyclic refractory major depressive disorder". J Affect Disord 1990, 20:193-200

51. Pande Ac, Calarco mm, Grunhaus LJ: "Combined MAOI-TCA treatment in refractory depression". IN Amsterdam J (ed): Refractory Depression . New York. Raven Press,1991,pp115-121.

52. Sethna ER: "A study of refractory cases of depressive illnes and their response to combined antidepressant treatment". Br J Psychiatry 1974, 124:265-272.

53. Fredman SJ, Fava M, Kienke AS, et al. "Partial response, nonresponse, and relapse with selective serotonin reuptake inhibitors in major depression: a survey of current next-step practices". J Clin Psychiatry 2000;61:403-408 
54. Muricio Fava MD: “Augmentation and Combination Strategies in Treatment-Resistant Depression". J Clin Psychiatry 2001;62(SUPPL 18) 4-11

55. Thase Me, Kupfer DJ, Frank E, et al: "Treatment of imipramine resistant recurrent depression: II _An open trial of lithium augmentation". J Clin Psychiatry 1989;50:413-417

56. Fink M: "A trial of ECT is essential before a diagnosis of refractory depression is made". In: Refractory depression. Amsterdam JD, ed, New York: Raven Press, 1991:87-92

57. Goodwin FK, Jamison KR. Manic-depressive illness. New York: Oxford University Press, 1990.

58. Price LH, Delgado PL, Charney DS, et al: "Sequence of drug administration in lithium augmentation: a case study". J Clin Psychiatry 1988;49:161-162

59. Lôo H, Venisse JL. “Le Lithium est-il un antidepresseur?” Rev Med 1978,19:433-436

60. Price LH, Charney DS, Geninher DR: "Efficacy of lithium-tranylcipromine treatment in refractory depression". Am J Psychiatry 1985;142:619-623

61. Ontiveros A, Fontaine R, Elie; R: "Refractory depression: the addition of lithium to fluoxetine or desipramine". Acta Psychiatr Scand 1991;83:188-192

62. Terao T, Yoshimura R, Abe K: "Lithium potentiation of tetracyclic antidepressant". Biol Psychiatry 1990;28:1075-77

63. Hoencamp E, Haffmans j, Dijken WA, Huijbrechts IP. "Lithium augmentation of venlafaxine: an open-label trial". J Clin Psychopharmacol 2000 Oct;20(5):538-43

64. Salama AA, Shafey M. "A case of severe lithium toxicity induced by combined fluoxetine and lithium carbonate" (letter) Am J Psychiatry 1989;146:278

65. Rybakowski JK, Suwalska A, Chlopocka M. "Potentiation of antidepressants with lithium or carbamazepine in treatment-resistant depression". Neuropsychobiology 1999 Sept;40(3):134-9

66. Malt UF, Fladvad T. "Lamotrigine in the tratment of mental disorders". Tidsskr Nor Laegeforen 2001;121 (12):1473-7

67. Yasmin S, Carpenter LL, Leon Z, Siniscalchi JM, Price LH. "Adjunctive gabapentin in treatment-resistant depression: a retrospective chart review". J Affect Disord 2001. 63(1-3):243-7

68. Prange AJ Jr. "Novel uses of thyroid hormones in patients with affective disorders". Thyroid 1996;6:537-543

69. Aronson R, Offman HJ, Joffe T, et al: "Triodothyronine augmentation in the treatment of the refractory depression: a meta-analysis". Arch Gen Psychiatry 1996;53:842-848

70. Targum SD, Greemberg RD, Harmon RL, et al: "The TRH test and thyroid hormone in refractory depression" (letter). Am J Psychiatry 1984; 141:463

71. Earle BV: "Thyroid hormone and trycyclic antidepressant in resistant depression". Am J Psychiatry 1970;126:143-145

72. Ostroff RB, Nelson JC. "Risperidone augmentation of selective serotonin reuptake inhibitors in major depression". J Clin Psychiatry 1999;60:256-259

73. Weizman R, Weizman A. "Use of atypical antipsychotics in mood disorders". Curr Opin Investig Drugs 2001 Jul;2(7):940-5

74. Shelton RC, Tollefson GD, Tohen M, et al. "A novel augmentation strategy for treating resistant major depression". Am J Psychiatry 2001;158:131-134

75. Glassman AH, Platman SR: "Potentiation of monoamine oxidase inhibitor by tryptophan". J Psychiatry Res 1969;7:83-88

76. Pare CMB: "Potentiation of monoamine oxidase inhibitors by tryptophan". Lancret 1963 II;527-528 
77. Berlanga C, Ortega-Soto HA, Ontiveros M, et al: "Efficacy of S-adenosyl-L-methionine in speeding the onset of action of imipramine". Psychiatry Res 1992;44:257-262

78. Rudorfer MV, Potter WZ: "Antidepressants: a comparative review of the clinical pharmacology and therapeutic use of the never versos the older drugs". Drugs 1989;37:713-718

79. Artigas F, Perez V, Alvarez E: "Pindolol induces a rapid improvement of depressed patients treated with serotonin reuptake inhibitors". Arch Gen Psychiatry 1994;51:248-251

80. Blier P, Bergeron R: "Effectiveness of pindolol with selected antidepressants drugs in the treatment of major depression". J Clin Pharmacol 1995;15:217-222

81. Olver JS, Cryan JF, Burrows GD, Norman Tr. "Pindolol augmentation of antidepressants: a review and rationale". Aust NZJ Psychiatry 2000;34(1):71-9

82. Joffe RT Schuller DR: "An open study of buspirone augmentation of serotonin reuptake inhibitors in refractory depression". J Clin Psychiatry 1993;54:269-271

83. Bakish D: "Fluoxetine potentiation by buspirone: three case histories". Can J Psychiatry 1991;36:749-750

84. Bakish D: "Fluoxetine potentiation by buspirone and trazodone in refractory depression". In Novel WA, Zohar J, Roose SP, Amsterdam JD (eds): Refractory depression: Current Strategies and Future Directions. Chichester, John Wiley \& Sons, 1994, pp143-145

85. Jacobsen FM: "Possible augmentation of antidepressant response by buspirone". J Clin Psychiatry 1991;52:217-220

86. Izumi T, Inoue T, Kitagawa N. "Open pergolide treatment of tricyclic and heterocyclic antidepressant-resistant depression". J Affect Disord 2000 Dec 1;61(1-2):127-132

87. Wharton R, Perel J, dayton P, etal. "A potential clinical use for methylphenidate with tricyclic antidepressants". Am J Psychiatry 1971;127:1619-1625

88. Nemets B, Mishory A, Levine J,et al. "Inositol addition does not improve depression in SSRI treatment failures". J Neural Transm. 1999;106:795-798

89. Levine J, Mishory A, Sunosky M, et al. "Combination of inositol and serotonin reuptake inhibitors in the treatment of depression". Biol Psychiatry 1999;45:270-273

90. Wolkowitz OM, Reus VI, Keebler A,etal.'Double blind treatment of major depression with dehydroepiandrosterone". Am J psychiatry 1999;156:646-649

91. Coppen A, Bailey J. "Enhancement of the antidepressant action of fluoxetine by folic acid: a randomised , placebo controlled trial". J Affect disord 2000;60:121-130

92. Bouwer C, Claassen J, Dinan TG, Nemeroff CB. "Prednisone augmentation in treatmentresistant depression with fatigue and hypocortisolaemia:a case series". Depress Anxiety 2000;12(1):44-50

93. Bodani M, Sheehan B, Philpot M. "The use of dexamethasone in elderly patients with antidepressant-resistant depressive illness". J Psychopharmacol 1999;13(2):196-7

94. Arana GW, Santos AB, Laraia MT, et al. "Dexamethasone for the treatment of depression: A randomized, placebo-controlled, double-bind trial". Am J Psychiatry 1995;152:265-267

95. Beale MD, Arana GW. "Dexamethasone for treatment of major depression in patients with bipolar disorder" (letter). Am J Psychiatry 1995;152:959-960

96. Amsterdam JD, Rosenzweig M, Mozley PD. "Assessment of adrenocortical activity in refractory depression: Steroid supression with Ketoconazole". In Nolen WA, Zohar J, Roose SP, Amsterdam JD (eds): Refractory Depression: Current Strategies and Future Directions. Chichester, John Wiley \& Sons, 1994, pp 199-210

97. Ravaris CL, Brinck-Johnsen C, Elliott B. "Clinical use of ketoconazole in hypocortisoluric depressives". Biol Psychiatry 1994;35:679. 
98. Thakore JH, Dina TG. "Cortisol synthesis inhibition: A new treatment strategy for the clinical and endocrine manifestations of depression". Biol Psychiatry 1995,37:364-368

99. Wolkowitz OM, Reus VI. "Treatment of depression with antiglucocorticoid drugs". Psychosom Med 1999 Sep-Oct;61(5):698-711

100. Bodkin JA, Lasser Ra, Wines JD Jr, et al. "Combining serotonin reuptake inhibitors and bupropion in partial responders to antidepressant monotherapy". J Clin Psychiatry 1997;58.137-145

101. Young SJ. "Panic associated with combining fluoxetine and bupropion" (letter). J Clin Psychiatry 1996;57:177-178

102. Labbate LA, Grimes JB, Hines A, et al. "Bupropion treatment of serotonin reuptake antidepressat-associated sexual dysfunction". Ann Clin Psychiatry 1997;9:241-245

103. Ashton AK, Rosen RC. "Bupropion as an antidote for serotonin reuptake inhibitor-induced sexual dysfunction”. J Clin Psychiatry 1998;59:112-115

104. Farah A. "Relief of SSRI-induced sexual dysfunction with mirtazapine treatment" (letter). J Clin Psychiatry 1999;60:260-261

105. Carpenter LL, Yasmin S, Hall JM, et al. "Mirtazapine augmentation in the treatment of refractory depression". J Clin Psychiatry 1999;60:45-49

106. Reynolds RD. "Sertraline-induced anorgasmia treated with intermittent nefazodone" (letter). J Clin Psychiatry 1997;58:89

107. Smith DL, Wenegrat BG. "A case report of serotonin syndrome associated with combined nefazodone and fluoxetine" (letter). J Clin Psychiatry 2000;61:146

108. John L, Perreault MM, Tao T, et al: "Serotonin syndrome associated with nefazodone and paroxetine". Ann Emerg Med 1997;29:287-289

109. Seth R, Jennings AL, Brindman J, et al. "Combined treatment with noradrenaline and serotonin reuptake inhibitors in resistant depression”. Br J Psychiatry 1992;161:562-565

110. Zajecka JM, Jeffries H, Fawcett J. "The efficacy of fluoxetine combined with heterocyclic antidepressant in treatment-resistant depression: a retrospective analysis". J Clin Psychiatry $1995 ; 56.3338-343$

111. Nelson JC, Mazure CM, Bowers MB, et al. "A preliminary open study of the combination of fluoxetine and desipramine for rapid treatment of major depression". Arch Gen Psychiatry 1991;48.303-307

112. Bondolfi G, Chautems C, Rochat B, et al. "Non-response to citalopram in depressive patients: pharmacokinetic and clinical consequences or a fluvoxamine augmentation" . Psychopharmacology (Berl) 1996;128:421-425

113. Gillman PK. "Serotonin syndrome. History and risk". Fun Clin Pharmacol 1998;12:482-491

114. Bhatara VS, Magnus RD, Paul KL, et al. "Serotonin syndrome induced by venlafaxine and fluoxetine: a case study in polypharmacy and potential pharmacodynamic mechanisms". Ann Pharmacother 1998;32:432-436

115. Benazzi F. "Venlafaxine-fluoxetine interaction”. J Clin Psychopharmacol 1999;19:96-98

116. Hawley CJ, Sivakumaran T, Ochocki M, et al. "Co-administration of reboxetine and serotonin selective reuptake inhibitors in treatment-resistant patients with major depression". $39^{\text {th }}$ annual meeting of the American College Neuropsychopharmacology; Dec 10142000. San Juan, Puerto Rico

117. Dursun Sm, Devarajan S. "The efficacy and safety of reboxetin plus citalopram in treatmentresistant depression: an open, naturalistic case series". $39^{\text {th }}$ annual meeting of the American College of Neuropharmacology; Dec 10-14, 2000. San Juan; Puerto Rico 
ORIGINALES Y REVISIONES

118. Fleishaker JC. "Clinical pharmacokinetics of reboxetine, a selective norepinephrine reuptake inhibitor for the treatment of patients with depression". Clin Pharmacokinet 2000;39:413-427

119. Madhukar H, Trivedi MD, Beverly PhD. "Using treatment algorithms for the effective management of treatment-resistant depression". J Clin Psychiatry 2001;62(suppl 18):25-28

120. Crismon ML, Trivedi MH, Pigott TA, et al. "The Texas Medication Algorithm Project: report of the Texas consensus conference panel on medication treatment of major depressive disorder". J Clin Psychiatry 1999;60:142-156

121. Star D: "Sequenced Treatment Alternatives to relieve Depression". Available at: www.edc.gsph.pitt.edu/stard. Accessed March 8, 2001

122. Trivedi MH, Kleiber BA. "Algorithm for the treatment of chronic depression". J Clin Psychiatry 2001;157:1501-1504

123. Trivedi MH,Kern JK, Baker SM,et al. "Computerized medical algorithms and decision support systems for major psychiatric disorders". J Clin Psychiatry 2000,6: 237-246

*Servicios de Salud Mental de Alcobendas. Madrid.

C/ Riscos de Polanco n1 14 61A. 28035 Madrid.

e-mail: lauragotor@inicia.es

Fecha de recepción: 3-V-02 